\title{
A metabolically engineered bacterium controls autoimmunity by remodeling the pro-inflammatory microenvironment
}

Jugal Kishore Das, \#, Fengguang Guo ${ }^{1,}$, Carrie Hunt ${ }^{2}$, Shelby Steinmeyer ${ }^{4}$, Julia A Plocica ${ }^{1}$, Koichi S. Kobayashi ${ }^{1,3}$, Arul Jayaraman ${ }^{4}$, Thomas A Ficht ${ }^{5, *}$, Robert C. Alaniz ${ }^{1, *}$, Paul de Figueiredo ${ }^{1,5, *}$, Jianxun Song, ${ }^{1 *}$

1 Department of Microbial Pathogenesis and Immunology, Texas A\&M University Health Science Center, Bryan, TX 77807, USA

${ }^{2}$ Department of Entomology, Texas A\&M University, College Station, TX 77845, USA

${ }^{3}$ Department of Immunology, Graduate School of Medicine, Hokkaido University, Sapporo 0608638, Japan

${ }^{4}$ Department of Chemical Engineering, Texas A\&M University, College Station, TX 77845, USA

${ }^{5}$ Department of Veterinary Pathobiology, Texas A\&M University, College Station, TX 77845, USA

\# These authors contributed equally to this work.

* To whom correspondence should be addressed (Dr Paul De Figueiredo; pjdefigueiredo@tamu.edu; Dr Robert C Alaniz; robert_alaniz@tamu.edu; Dr Thomas A Ficht; tficht@tamu.edu; Dr Jianxun Song; jus35@tamu.edu) 


\begin{abstract}
Immunotherapy has led to impressive advances in the treatment of autoimmune and proinflammatory disorders; yet, its clinical outcomes remain limited by a variety of factors including the pro-inflammatory microenvironment (IME). Discovering effective immunomodulatory agents, and the mechanisms by which they control disease, will lead to innovative strategies for enhancing the effectiveness of current immunotherapeutic approaches. We have metabolically engineered an attenuated bacterial strain (i.e., Brucella melitensis $16 \mathrm{M} \Delta v j b R, \mathrm{Bm} \Delta v j b R$ ) to produce indole, a tryptophan metabolite that controls the fate and function of regulatory $\mathrm{T}$ cells ( $\left.\mathrm{T}_{\text {regs }}\right)$. We demonstrated that treatment with this strain polarized M2 macrophages $(\mathrm{M} \varphi)$ which produced anti-inflammatory cytokines (e.g., IL-10) and promoted $\mathrm{T}_{\text {reg }}$ function; moreover, when combined with adoptive cell transfer $(\mathrm{ACT})$ of $\mathrm{T}_{\text {regs, }}$ a single treatment with our engineered bacterial strain dramatically reduced the incidence and score of autoimmune arthritis and decreased joint damage. These findings show how a metabolically engineered bacterium can constitute a powerful vehicle for improving the efficacy of immunotherapy, defeating autoimmunity and reducing inflammation by remodeling the IME and augmenting $\mathrm{T}_{\text {reg }}$ function.
\end{abstract}




\section{Main}

Numerous studies have demonstrated promising results using immunotherapy to treat autoimmune and pro-inflammatory disorders ${ }^{1-4}$. Nevertheless, despite advances in the field of immunotherapy, including regulatory $\mathrm{T}$ cell $\left(\mathrm{T}_{\text {reg }}\right)$-based therapy, the efficacy and benefits of these approaches remain less satisfactory due to limited in vivo $\mathrm{T}_{\text {reg }}$ expansion and persistence, the pro-inflammatory microenvironment (IME), and insufficient $\mathrm{T}_{\text {reg }}$ trafficking to inflamed sites. In addition, immune dysregulation of the IME contributes to disease ${ }^{5}$. Immunotherapeutic combinations may produce greater efficacy, and thus strategies that circumvent these barriers are urgently needed.

To address this need, we developed and tested an intervention that combined two innovations. First, although a therapeutic role for live attenuated bacterial vaccines in addressing infectious diseases is undeniable and appreciated in cancer immunotherapy, the use of bacterial agents to manage autoimmune and pro-inflammatory diseases remains limited. Second, a growing number of microbiota-specific products and metabolites have novel immunologic properties that constitute one mechanism whereby the microbiota influence host health and disease ${ }^{6,7}$. We therefore developed a metabolically engineered bacterial vaccine to produce immunomodulatory metabolites that improve autoimmunity and inflammation.

For the bacterial vector, we selected an attenuated strain of Brucella melitensis that harbors a deletion in $v j b R$, a master regulator of virulence $(\mathrm{Bm} \Delta v j b R)^{8}$. Like other Gram-negative organisms, Brucella strains express a lipopolysaccharide (LPS) lacking endotoxin activity. Importantly, Bm $\Delta v j b R$ has been shown to be safe in immunocompetent and 
immunocompromised mice ${ }^{9}$, goats ${ }^{10}$, sheep ${ }^{11}$, and non-human primates $^{12}$. We also have shown that $\mathrm{Bm} \Delta v j b R$ can combat cancer in a murine model by remodeling the tumor microenvironment (TME) to a pro-inflammatory state ${ }^{8}$. Moreover, when $\mathrm{Bm} \Delta v j b R$ treatment was combined with adoptive cell transfer (ACT) of tumor antigen (Ag)-specific $\mathrm{CD}^{+} \mathrm{T}$ cells, tumor growth and proliferation were dramatically impaired ${ }^{8}$. Conversely, in the current studies, we engineered $\mathrm{Bm} \Delta v j b R$ to express tryptophanase (tnaA); i.e., Bm $\Delta v j b R::$ tnaA, to produce the tryptophan metabolite indole, a molecule that modulates the fate and function of $\mathrm{T}_{\text {regs }}{ }^{13}$.

We have reported that indole, when used at a range of physiologic concentrations, suppresses several inflammatory characteristics in immune and non-immune cells, and also augments $\mathrm{T}_{\text {reg }}$ differentiation ${ }^{14}$. Consistent with our previous reports, we demonstrated that indole suppressed TNF- $\alpha$ production in $\mathrm{CD}_{1} 1 \mathrm{~b}^{+}$spleen cells after $E$ coli LPS (eLPS) stimulation (Fig. 1a \& Fig. 1b) and dampened their activation by suppressing Akt and ERK signaling pathways in response to microbial agonists (eLPS and heat-inactivated Salmonella Typhimurium [HKST]) (Fig. S1a). In addition, indole augmented the differentiation of naive $C D 4^{+} \mathrm{CD} 25^{-} \mathrm{T}$ cells into induced $\mathrm{T}_{\text {regs }}$ (iT $\left.\mathrm{T}_{\text {regs }}\right)$ measured by FoxP3 in vitro in a dose dependent manner (Fig. 1c \& Fig. S1f). These findings were consistent with our earlier reports ${ }^{11}$, and were comparable to results from studies using the microbiota metabolite butyrate, albeit with distinct dose-dependency ${ }^{15}$. Based on these findings, we hypothesized that indole would ameliorate immune-mediated inflammation in autoimmune and pro-inflammatory diseases.

Several lines of investigation demonstrated that indole reduces autoimmune responses in a murine collagen-induced arthritis (CIA) model. First, we showed that the severity of CIA was 
significantly attenuated in indole treated mice, which exhibited clinical scores of $0.8 \pm 0.2$ (means \pm SEM) at (Day 50), compared to $1.6 \pm 0.5$ in controls (Fig. 1d). However, a single dose of indole only showed a slight decrease in inflammation. Similarly, single dose treatment did not induce significant alterations in the infiltration of $\mathrm{T}_{\text {regs }}$ as assessed by confocal microscopy (Fig. 1e \& Fig. 1f). These findings were in striking contrast to our ex vivo experimental findings, which indicated that indole significantly promoted the expansion of $\mathrm{CD}^{+} \mathrm{FoxP} 3^{+} \mathrm{T}_{\text {regs }}$ and enhanced their activation by increased expression of PD-1 compared to the controls $(p<0.001)$, an immunosuppressive molecule, in cells derived from the mouse lymph nodes (LNs) and spleen (Fig. 1g). Based on our in vivo and ex vivo findings, we hypothesized that the sustained delivery of indole in a bacterial vector may greatly improve the durability of the molecule's immunomodulatory effects, resulting in an attenuation of autoimmunity and inflammation in CIA. To test this hypothesis, we engineered a safe live-attenuated bacterial strain (Bm $\Delta v j b R:: \operatorname{tna} A)$ to constitutively produce indole (Fig. S1c, Fig. S1d \& Fig. S1e).

First, cytokine array profiling analyses showed that Bm $\Delta v j b R:: t n a A$ induced the expression of IL-10 (Fig. 2a \& Fig. S2a), which promotes the activities of $\mathrm{T}_{\text {regs }}$ and reduces autoimmunity and inflammation $^{16,17}$. Strikingly, Bm $\Delta v j b R::$ tnaA also significantly $(p<0.01)$ reduced the expression of additional pro-inflammatory cytokines like IL-6, IL-1 $\beta$ and TNF- $\alpha$ in macrophages (M $\varphi$ ) compared to Bm $\Delta v j b R$ parental strain (Fig. 2a). Second, we found that Bm $\Delta v j b R:: t n a A$, when co-cultured with bone marrow-derived $\mathrm{M} \varphi$ (BMDMs), not only significantly reduced the total $\mathrm{CD}^{+} \mathrm{T}$ cells $(p<0.001)$ but also reduced the production of the pro-inflammatory cytokines such as TNF- $\alpha$ and IFN- $\gamma(p<0.001)$ compared to the Bm $\Delta v j b R$ parental strain (Fig. 2b). Moreover, $\mathrm{Bm} \Delta v j b R::$ tnaA promoted the expansion of $\mathrm{T}_{\mathrm{regs}}$ and significantly enhanced their activity as 
assessed by IL-10 production $(p<0.001)$ and PD-1 expression $(p<0.01)$ (Fig. S2a). Third, in the CIA model, a significant reduction in arthritis score and incidence was observed following treatment with Bm $\Delta v j b R::$ tnaA. This amelioration of autoimmunity and inflammation was further augmented when Bm $\Delta v j b R::$ tnaA treatment was combined with ACT of $\mathrm{T}_{\text {regs }}$ (Fig. 2c). Fourth, we observed significantly $(p<0.01)$ reduced numbers of infiltrating inflammatory cells into the joints of mice treated with $\mathrm{Bm} \Delta v j b R::$ tnaA. This effect was further enhanced by $\mathrm{Bm} \Delta v j b R::$ tnaA treatment followed with ACT of $\mathrm{T}_{\text {regs }}$ compared to the controls $(p<0.001)$ (Fig. 2d). Finally, mice treated with Bm $\Delta v j b R::$ tnaA showed reduced infiltrates in the joint as evidenced by H\&E analysis and Safranin O (Saf-O) staining of knee cross-sections (60 days post collagen administration). Notably, these findings were further attenuated by addition of ACT of $\mathrm{T}_{\text {regs }}$ (Fig. 2d). There was also a significant reduction in the total $\mathrm{CD}^{+} \mathrm{T}$ cell proportions and a dramatic increase in $\mathrm{T}_{\text {reg }}(p<0.001)$ proportions in mice treated with $\mathrm{Bm} \Delta v j b R: \because$ tnaA compared to controls (Fig. 2e).

To identify the mechanism by which Bm $\Delta v j b R::$ tnaA might be acting, we conducted a multiparametric CyTEK analysis from the cells isolated from the joints of control, ACT with $\mathrm{T}_{\text {reg }}$ only, or ACT with $\mathrm{T}_{\text {reg }}$ plus Bm $\Delta v j b R::$ tnaA groups. We found the Bm $\Delta v j b R::$ tnaA reduced the proportion of B cells (Fig. 2f, 2g, and Table S1) in addition to promoting $\mathrm{T}_{\text {reg }}$ expansion. Overall, our results indicate that $\mathrm{Bm} \Delta v j b R::$ tnaA not only remodels the IME and facilitates the expansion and suppressive function of $\mathrm{T}_{\text {regs }}$ but may also modulate $\mathrm{B}$ cell-mediated immunity in the CIA model. 
Several strategies have been employed to enhance the efficacy of live attenuated bacterial vaccines. However, bacterial vaccines producing immunomodulatory metabolites that alter the immunological tolerance and the IME have not been previously reported. Our work features both conceptual and methodological innovations. First, this work provides the first description of a live attenuated vaccine whose metabolism has been reprogrammed to amplify antiautoimmune/inflammation activity. Second, this study provides the first description of how combining a single dose of a live attenuated bacterial vaccination with ACT of $\mathrm{T}_{\text {regs }}$ can achieve potent therapeutic outcomes. Third, we demonstrate how the genetic tractability of Bm $\Delta v j b R$ can be exploited to engineer vaccines with novel properties. Although in this work, we focused on metabolic reprogramming of the bacterium, we envision that future iterations of the Bm $\Delta v j b R::$ tnaA vaccine will include engineered auto Ags to boost persistent auto Ag-specific $\mathrm{T}_{\text {reg }}$ responses. Fourth, this work showed that indole did not succeed as as a stand-alone agent. However, this limitation was circumvented by delivery using our engineered bacterial vector. Bm $\Delta v j b R::$ tnaA may offer improved pharmacodynamics for indole by sustained production levels and effects in vivo versus indole alone. Morever, we showed that the separate individual beneficial effects of $\mathrm{Bm} \triangle v j b R$ and indole act synergistically in vivo. Finally, the natural localization of $\mathrm{Bm} \triangle v j b R$ to leukocytes enabled $\mathrm{Bm} \Delta v j b R:: \operatorname{tnaA}$ to provide improved targeted indole delivery and a higher local effective indole concentration in contrast to the untargeted and transient nature of indole as a single agent. In sum, our work presents an attractive new avenue for the development of anti-autoimmunity/inflammation vaccines. 


\section{Methods}

\section{Immunization with CIA}

CIA was induced as described previously with minor modifications ${ }^{18}$. Briefly, male C57BL/6 mice were injected with an emulsion of $100 \mu \mathrm{l}$ of chick type II collagen (Chondrex; $100 \mu \mathrm{g})$ in Complete Freund's Adjuvant (CFA; Chondrex) using a glass tuberculin syringe with 26-gauge needle. The mice were then assessed for development of joint inflammation and clinical arthritis score until Day 60.

\section{Bacterial culture}

$\mathrm{Bm} \Delta v j b R$ or $\mathrm{Bm} \Delta v j b R:: \operatorname{tnaA}$ were cultivated and prepared for experimentation as previously described $^{8}$.

\section{Engineering indole-producing $B m \Delta v j b R:: t n a A$ strain}

To generate an indole producing attenuated $\mathrm{Bm} \Delta v j b R$ strain, we cloned an Escherichia coli $(E$. coli) thaA gene into a broad range bacteria expression plasmid (pBBR1MCS6Y) ${ }^{19}$ and transferred the plasmid to $\mathrm{Bm} \Delta v j b R$.

\section{BmSvjbR::tnaA treatment}

CIA was induced in male C57BL/6 mice. On Day 7 after the CIA induction, mice were i.v. injected with $5.0 \times 10^{7}$ live $\mathrm{Bm} \Delta v j b R::$ tnaA or PBS control. In the Bm $\Delta v j b R::$ tnaA $+\mathrm{T}_{\text {reg }}$ combinatorial treating experiments, mice $(\mathrm{N}=5)$ were adoptively transferred with $2.5 \times 10^{6}$ $\mathrm{CD}^{+} \mathrm{CD} 25^{+} \mathrm{T}_{\text {regs }}$ derived from donor LNs and spleen of naive C57BL/6 mice, one week after the $\mathrm{Bm} \Delta v j b R::$ tnaA administration. 


\section{Flow cytometric analysis}

Cell staining and flow cytometric analysis were performed as described previously ${ }^{8}$ using the described labeling reagents. Briefly, surface and intracellular staining was performed on the single-cell suspensions and analyzed using LSR Fortessa cell analyzer (BD). The joints were also processed and stained similarly with atibodoies listed in Table S2, and data was acquired on CyTEK aurora flowcytometer (Cytek Biosciences). For multiparametric analyses, the data were analyzed with FlowJo v10 and represented as heatmaps and tSNE plots.

\section{Histology and immunofluorescence}

Mice were humanely sacrificed on day 60 after induction of CIA, and tissue sections were analyzed as previously described ${ }^{18}$. Briefly, the hind foot paws and knees were removed and fixed in 10\% formalin and decalcified in Formical-4 (Decal chemical, Tallman, NY). The fixed tissue sections were then stained with H\&E and/or Safranin O fast green (Saf-O) stain. The H\&E and Saf-O stained sections were then assessed by semiquantitative system of 0 to 4 as described previously.

Immunofluorescent staining and microscopy were performed on the deparaffinized sections by using FITC anti-mouse FoxP3 antibody $(\mathrm{Ab})$ for $\mathrm{T}_{\text {regs }}$ and DAPI as nuclear stain.

\section{Statistical Analysis}

Student's $t$-test was performed for statistical analysis between the groups. All analyses were performed in GraphPad Prism v9. A $p$ value of $<0.05$ was considered statistically significant. 


\section{AUTHORS' CONTRIBUTIONS}

JS, PDF, RCA and TF conceived and designed the experiments. JKD and FG performed the experiments and analyzed the data. JS, PDF, JKD, FG, RCA, TF, CH, SH, JAP, KSK and AJ wrote the manuscript and provided critical feedback. JS and PDF supervised the research. All the authors read and approved the final manuscript.

\section{ACKNOWLEDGMENTS}

We thank Robbie Moore from COM-CAF, Malea Murphy from IMIL, Sankar P. Chaki from TAMH-CVM, and Elizabeth Bustamante from TAMU-HSC, and the Immunomonitoring Core facility at Houston Methodist Hospital-Texas Medical Center for their technical supports. This work was supported by funding from NIH (R01AI121180 and R01CA221867 to J.S., R01AI110642 to R.A., R01HD084339 to T.A.F., and R01AI141607-01A1 to P.D.F.), NSF DBI1532188, and NSF0854684 to P.D.F. 


\section{FIGURE LEGENDS}

Fig. 1: Indole treatment dampens inflammation and promotes $T_{\text {reg }}$ expansion and activity. a, Indole reduces production of pro-inflammatory TNF- $\alpha$ in CD11 b cells. $0.25,0.5$, or $1.0 \mathrm{mM}$ Indole was dissolved in DMF for the representative experimental flowcytometric analysis. b, Graphical representation of flow cytometric dot-plots derived from 3 independent experiments of HKST group. c, Indole promotes dose-dependent differentiation of $\mathrm{T}_{\text {regs; }}$ Experiment $(\mathrm{N}=3)$ was performed under $T_{\text {reg }}$ skew conditions $([$ TGF- $\beta]=2 \mathrm{ng} / \mathrm{mL},[\mathrm{IL}-2]=100 \mathrm{U} / \mathrm{mL}) . \mathrm{Th}_{0}$ control represents non- $\mathrm{T}_{\text {reg }}$ skew conditions. Butyrate was used as a control metabolite. $\mathrm{d}$, Effects of indole alone on CIA in mice $(\mathrm{N}=5)$. e, Representative images of H\&E, Safranin O (Saf-O) stained tissues, and confocal microscopy of knee tissues of CIA mice on day 60 post induction of arthritis. f, Quantitative analysis of $\mathrm{H} \& \mathrm{E}, \mathrm{Saf}-\mathrm{O}$ and $\mathrm{T}_{\text {reg }}$ infiltration from confocal microscopy sections of Control (Ctrl) and indole-treated mice. g, Flow cytometric dot-plot analysis of PD-1 and FoxP3 in indole-treated, ex vivo stimulated $\mathrm{CD}^{+} \mathrm{T}$ cells derived from the LNs and spleen and activated with anti-CD3/CD28 Abs. Exposure to indole drives these cells towards higher $\mathrm{T}_{\text {reg }}$ phenotype by increased FoxP3 expression. Graphical representation of CD4 ${ }^{+} \mathrm{T}$ cells $(\%)$ derived from the flow cytometric dot-plots of $\mathrm{CD}^{+} \mathrm{T}$ cells exposed to indole. Graphical representation of $\mathrm{CD}^{+} \mathrm{FoxP}^{+} \mathrm{PD}-1^{+} \mathrm{T}$ cells $(\%)$ from the flow cytometric dot-plots. Data represent means \pm SD. Student's $t$-test or Tukey's multiple comparisons test was applied for statistical analysis. *, $* *, * * *$ : significance at $\mathrm{p}<0.05,0.01,0.001$

Fig. 2: Bm $\triangle v j b R:: t n a A$ significantly dampens inflammation and reduces arthritis in murine CIA model which is augmented by adoptive cell transfer (ACT) of $\mathbf{T}_{\text {regs. }}$ 
a, Cytokine arrays were used to measure pro-inflammatory cytokines produced by control, $\mathrm{Bm} \triangle v j b R$, and Bm $\Delta v j b R::$ tnaA treated BMDMs. b, BMDMs were treated with either $\mathrm{Bm} \Delta v j b R::$ tnaA or Bm $\Delta v j b R$ and then co-cultured with $\mathrm{T}$ cells derived from pooled LNs and spleen of C57BL/6 mice. Flow cytometric assays were then performed to measure IFN- $\gamma$ and TNF- $\alpha$ of T cells. c, Arthritis score and arthritis incidence in CIA C57BL/6 mice from control (Ctrl, gray bar); Bm $\triangle v j b R$ (blue bar); $\mathrm{Bm} \Delta v j b R::$ tnaA (pink bar) and Bm $\Delta v j b R::$ tnaA followed by ACT of $\mathrm{T}_{\text {regs }}\left(\mathrm{T}_{\text {reg, }}\right.$, red bar) $(\mathrm{N}=5$ in each group). $\mathrm{d}$, Representative images of H\&E, Saf-O staining, and confocal microscopy from mouse knees on day 60 post CIA induction. Quantitative analysis of $\mathrm{T}_{\text {reg }}$ infiltration and inflammation scores from these mice are also shown. e, Cells from the LNs and spleen were collected from CIA-induced mouse groups (Ctrl, Bm $\Delta v j b R:: t n a A$, and Bm $\Delta v j b R::$ tnaA combined with ACT of $\mathrm{T}_{\text {regs. }}$ These cells were then stained and quantified by flow cytometry using markers for $\mathrm{CD}^{+} \mathrm{T}$ cells and intracellular staining of FoxP3 $\left(\mathrm{T}_{\text {regs }}\right)$. $\mathrm{f}$, CIA-induced mice were treated with PBS (Ctrl), ACT of $\mathrm{T}_{\text {regs }}$ only $\left(\mathrm{T}_{\text {regs }}\right.$ only; $\left.\mathrm{N}=5\right)$, or $\mathrm{Bm} \Delta v j b R::$ tnaA combined with ACT of $\mathrm{T}_{\text {regs }}(\mathrm{N}=5)$. Cells from the knee and ankle joints were stained with 21 markers and measured by CyTEK aurora flow cytometry. Heatmap shows immune cell profiles in different treatment groups of mice (scale bar represents percentage of cell in each treatment group within each cell type). g, viSNE map shows the four subtypes of B cells differentially expressed in the treated group of mice. Data represent means \pm SD. Student's $t$-test or Tukey's multiple comparisons test was applied for statistical analysis. *, **, ***: significance at $\mathrm{p}<0.05,0.01,0.001$. 


\section{Supplementary Figures}

Fig. S1: Indole suppresses immune cell activation and $\mathrm{Bm} \triangle \mathrm{vjBR}$ is engineered to produce indole.

a, Indole $(1 \mathrm{mM})$ suppresses the Akt and ERK signaling pathways in CD11 $\mathrm{b}^{+}$cells in different post incubation time. Flow cytometric dot-plot analysis and graphical representation of Akt and ERK expression with time. b, Schematic diagram of murine CIA model used in all the subsequent studies. c, Schematic representation of the engineered Bm $\Delta v j b R:$ tnaA harboring a plasmid carrying a tnaA expression cassette. The indole biosynthesis pathway is depicted in the figure. TnaA catalyzes the conversion of tryptophan to indole. d, Engineered Bm $\Delta v j b R:: \operatorname{tnaA}$ produced high concentration of indole as shown in the mass-spectrometric analysis. e, Western blotting analysis of the expression of tnaA protein in the parental strain compared with the engineered Bm $\Delta v j b R:: \operatorname{tna} A$ strain. Graphical representation of the comparative analysis of indole production by $\mathrm{Bm} \triangle v j B R$ parental bacterial strain and the engineered $\mathrm{Bm} \Delta v j b R:: \operatorname{tnaA}$ strain. $\mathrm{f}$, Indole promotes significantly higher expansion of $\mathrm{T}_{\mathrm{regs}}$ compared to butyrate at $1 \mathrm{mM}$ concentration as shown in the graphical analysis. Graphical data derived from flow cytometric representatives as shown in Fig. 1C. Data represent means \pm SD. Student's $t$-test or Tukey's multiple comparisons test was applied for statistical analysis. *,**,***: significance at $p<0.05$, $0.01,0.001$

Fig. S2: Bm $\Delta v j b R:: t n a A$ activates $\mathrm{T}_{\text {regs }}$ and suppresses B-cell mediated inflammation.

a, BMDMs infected with either Bm $\Delta v j b R:: t n a A$ or Bm $\Delta v j b R$ were co-cultured with $\mathrm{CD}^{+} \mathrm{T}$ cells from mouse LNs and spleen and activated by using anti-CD3/CD28 Abs. Flow cytometric dotplot assay shows that Bm $\Delta v j b R::$ tnaA treated BMDMs greatly promoted expression of FoxP3 
and PD-1 and production of IL-10 in $\mathrm{CD}^{+} \mathrm{T}$ cells. The dot-plots are followed by graphical representation from 3 independent experiments. b, The total CD19 population is shown in the viSNE plots. Data represent means \pm SD. Student's $t$-test or Tukey's multiple comparisons test was applied for statistical analysis. $* * *, * * *$ : significance at $p<0.05,0.01,0.001$. 


\section{References}

1. Roth, T.L., et al. Pooled Knockin Targeting for Genome Engineering of Cellular Immunotherapies. Cell 181, 728-744 e721 (2020).

2. Kansal, R., et al. Sustained B cell depletion by CD19-targeted CAR T cells is a highly effective treatment for murine lupus. Sci Transl Med 11(2019).

3. Dall'Era, M., et al. Adoptive Treg Cell Therapy in a Patient With Systemic Lupus Erythematosus. Arthritis Rheumatol 71, 431-440 (2019).

4. Bluestone, J.A., et al. Type 1 diabetes immunotherapy using polyclonal regulatory $\mathrm{T}$ cells. Sci Transl Med 7, 315 ra189 (2015).

5. Sumida, T., et al. Activated beta-catenin in Foxp3(+) regulatory $\mathrm{T}$ cells links inflammatory environments to autoimmunity. Nat Immunol 19, 1391-1402 (2018).

6. Han, S., et al. A metabolomics pipeline for the mechanistic interrogation of the gut microbiome. Nature 595, 415-420 (2021).

7. Henrick, B.M., et al. Bifidobacteria-mediated immune system imprinting early in life. Cell 184, 3884-3898 e3811 (2021).

8. Guo, F., et al. Live attenuated bacterium limits cancer resistance to CAR-T therapy by remodeling the tumor microenvironment. Journal for immunotherapy of cancer 10(2022).

9. Costa, L.F., et al. The candidate vaccine strain Brucella ovis abcBA is protective against Brucella melitensis infection in mice. Microbiol. Immunol. 64, 730-736 (2020).

10. Castano-Zubieta, M.R., et al. Evaluation of the safety profile of the vaccine candidate Brucella melitensis 16MDeltavjbR strain in goats. Vaccine 39, 617-625 (2021).

11. Hensel, M.E., et al. Vaccine Candidate Brucella melitensis 16MDeltavjbR Is Safe in a Pregnant Sheep Model and Confers Protection. mSphere 5(2020).

12. Russell-Lodrigue, K.E., Killeen, S.Z., Ficht, T.A. \& Roy, C.J. Mucosal bacterial dissemination in a rhesus macaque model of experimental brucellosis. J. Med. Primatol. 47, 75-77 (2018).

13. Steinmeyer, S., Howsmon, D.P., Alaniz, R.C., Hahn, J. \& Jayaraman, A. Empirical modeling of $\mathrm{T}$ cell activation predicts interplay of host cytokines and bacterial indole. Biotechnol. Bioeng. 114, 2660-2667 (2017).

14. Krishnan, S., et al. Gut Microbiota-Derived Tryptophan Metabolites Modulate Inflammatory Response in Hepatocytes and Macrophages. Cell Rep 28, 3285 (2019).

15. Furusawa, Y., et al. Commensal microbe-derived butyrate induces the differentiation of colonic regulatory T cells. Nature 504, 446-450 (2013).

16. Tanaka, S., et al. Trim33 mediates the proinflammatory function of Th17 cells. $J$ Exp Med 215, 1853-1868 (2018).

17. Ramakrishna, C., et al. Bacteroides fragilis polysaccharide A induces IL-10 secreting B and T cells that prevent viral encephalitis. Nature communications 10, 2153 (2019).

18. Haque, R., et al. Programming of regulatory $\mathrm{T}$ cells from pluripotent stem cells and prevention of autoimmunity. J Immunol 189, 1228-1236 (2012).

19. Fernandez-Prada, C.M., et al. Interactions between Brucella melitensis and human phagocytes: bacterial surface O-Polysaccharide inhibits phagocytosis, bacterial killing, and subsequent host cell apoptosis. Infect. Immun. 71, 2110-2119 (2003). 
Fig. 1: Indole treatment dampens inflammation and promotes $T_{\text {reg }}$ expansion and activity. a, Indole reduces production of pro-inflammatory TNF- $\alpha$ in CD11 $b^{+}$cells. $0.25,0.5$, or $1.0 \mathrm{mM}$ Indole was dissolved in DMF for the representative experimental flowcytometric analysis. b, Graphical representation of flow cytometric dot-plots derived from 3 independent experiments of HKST group. c, Indole promotes dose-dependent differentiation of $\mathrm{T}_{\text {regs }}$; Experiment ( $\mathrm{N}=3)$ was performed under $\mathrm{T}_{\text {reg }}$ skew conditions $([T \mathrm{GF}-\beta]=2 \mathrm{ng} / \mathrm{mL}$, [IL-2] $=100$ $\mathrm{U} / \mathrm{mL}$ ). $\mathrm{Th}_{0}$ control represents non- $\mathrm{T}_{\text {reg }}$ skew conditions. Butyrate was used as a control metabolite. d, Effects of indole alone on $\mathrm{ClA}$ in mice (N=5). e, Representative images of $\mathrm{H} \& \mathrm{E}$, Safranin O (Saf-O) stained tissues, and confocal microscopy of knee tissues of CIA mice on day 60 post induction of arthritis. f, Quantitative analysis of H\&E, Saf-O and $T_{\text {reg }}$ infiltration from confocal microscopy sections of Control (Ctrl) and indole-treated mice. g, Flow cytometric dot-plot analysis of PD-1 and FoxP3 in indole-treated, ex vivo stimulated CD4 ${ }^{+} \mathrm{T}$ cells derived from the LNs and spleen and activated with anti-CD3/CD28 Abs. Exposure to indole drives these cells towards higher $\mathrm{T}_{\text {reg }}$ phenotype by increased FoxP3 expression. Graphical representation of $\mathrm{CD}^{+} \mathrm{T}$ cells (\%) derived from the flow cytometric dot-plots of $\mathrm{CD} 4^{+} \mathrm{T}$ cells exposed to indole. Graphical representation of $\mathrm{CD} 4^{+} \mathrm{FoxP} 3^{+} \mathrm{PD}-1^{+} \mathrm{T}$ cells $(\%)$ from the flow cytometric dot-plots. Data represent means $\pm \mathrm{SD}$. Student's $t$-test or Tukey's multiple comparisons test was applied for statistical analysis. ${ }^{*},{ }^{* *},{ }^{* * *}$ : significance at $p<$ $0.05,0.01,0.001$. 


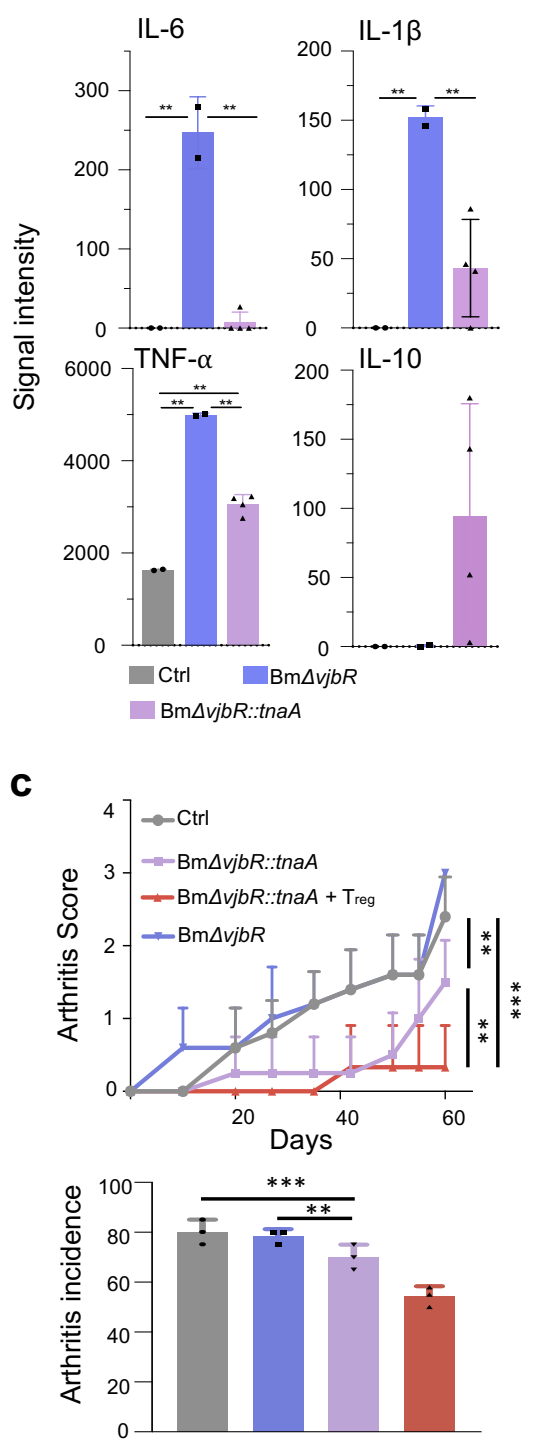

b
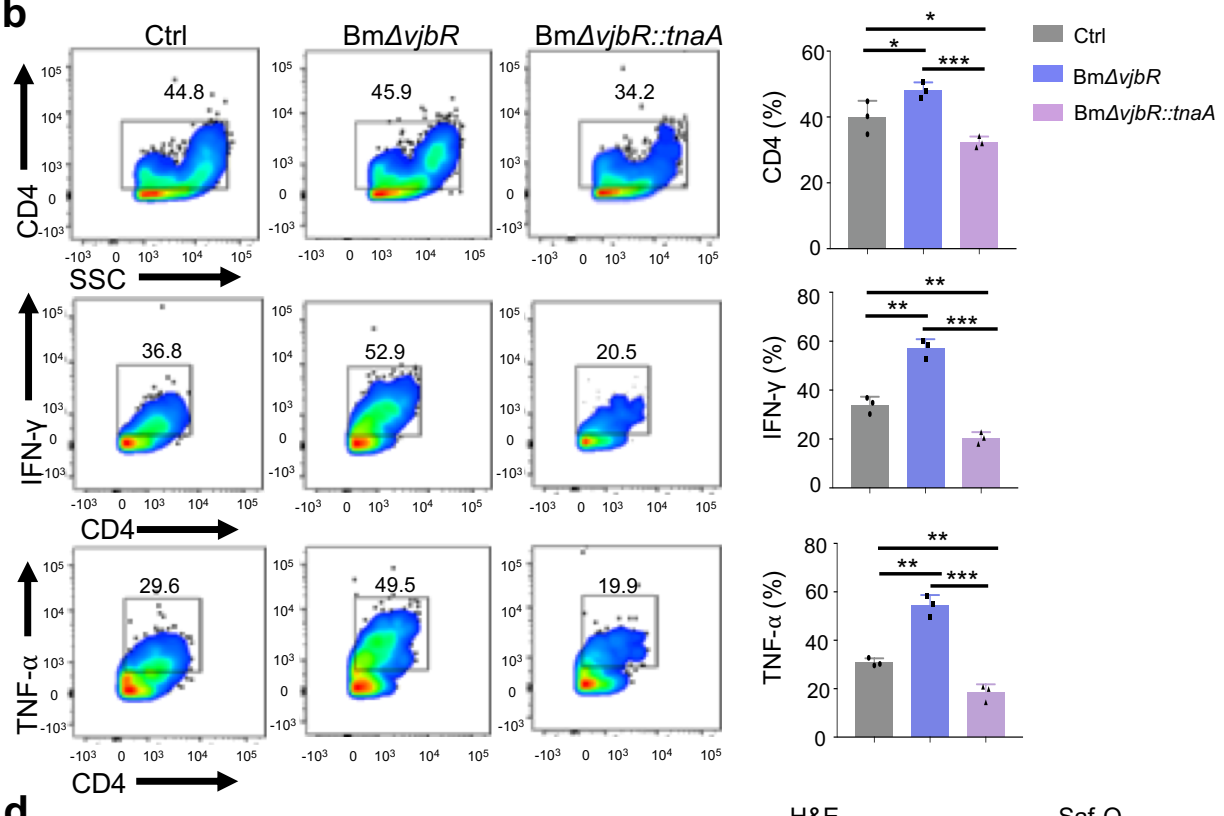

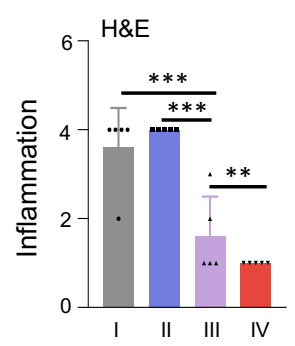

6 Saf-O
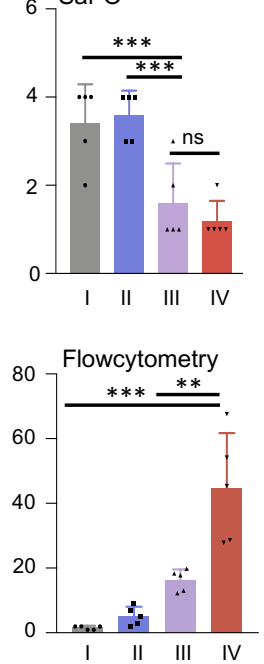

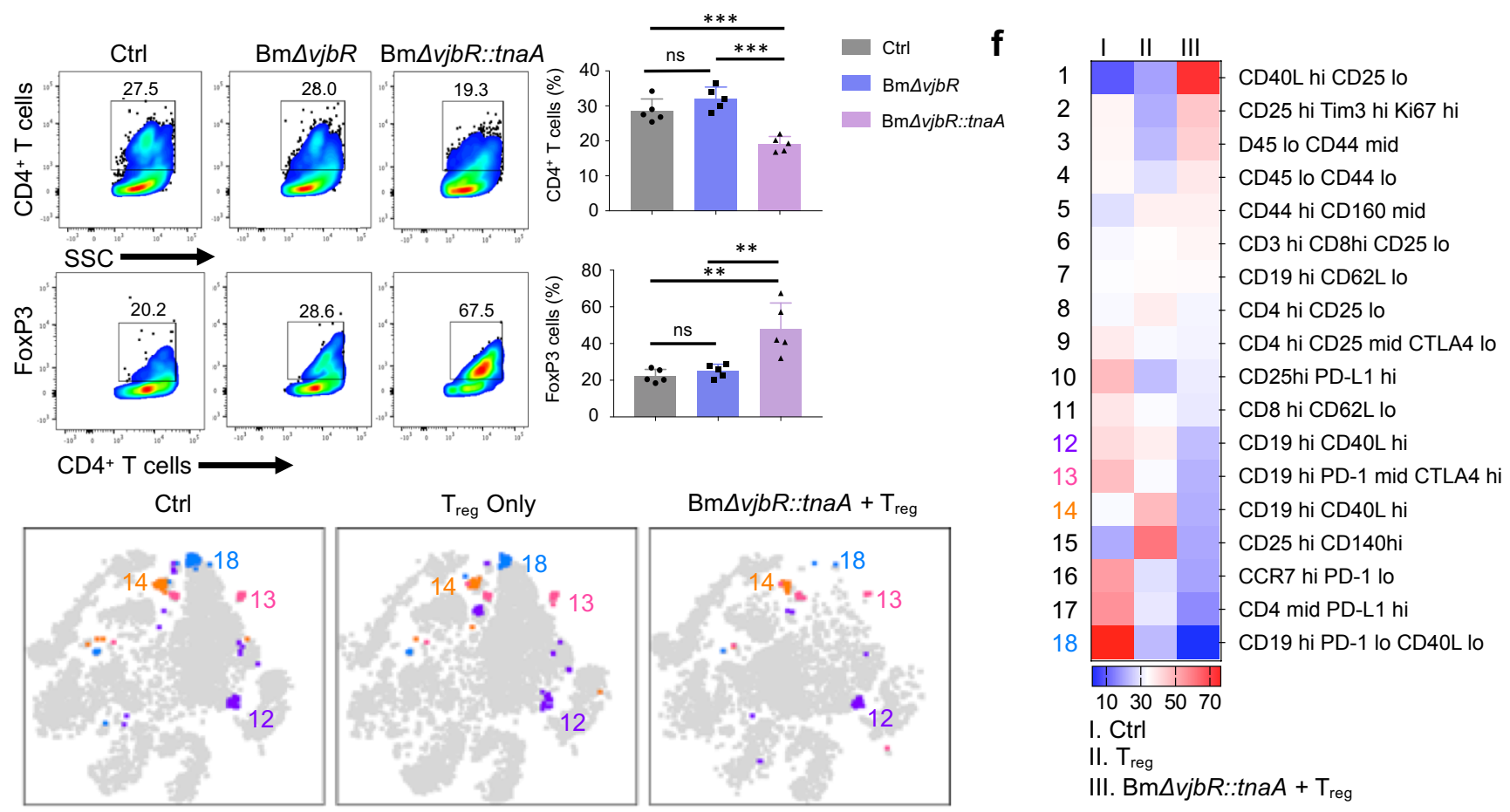

g

e d

I. Ctrl II. Bm $\Delta v j b R$

III. Bm $\Delta v j b R:: t n a A \quad$ IV. Bm $\Delta v j b R:: t n a A+\mathrm{T}_{\text {reg }}$
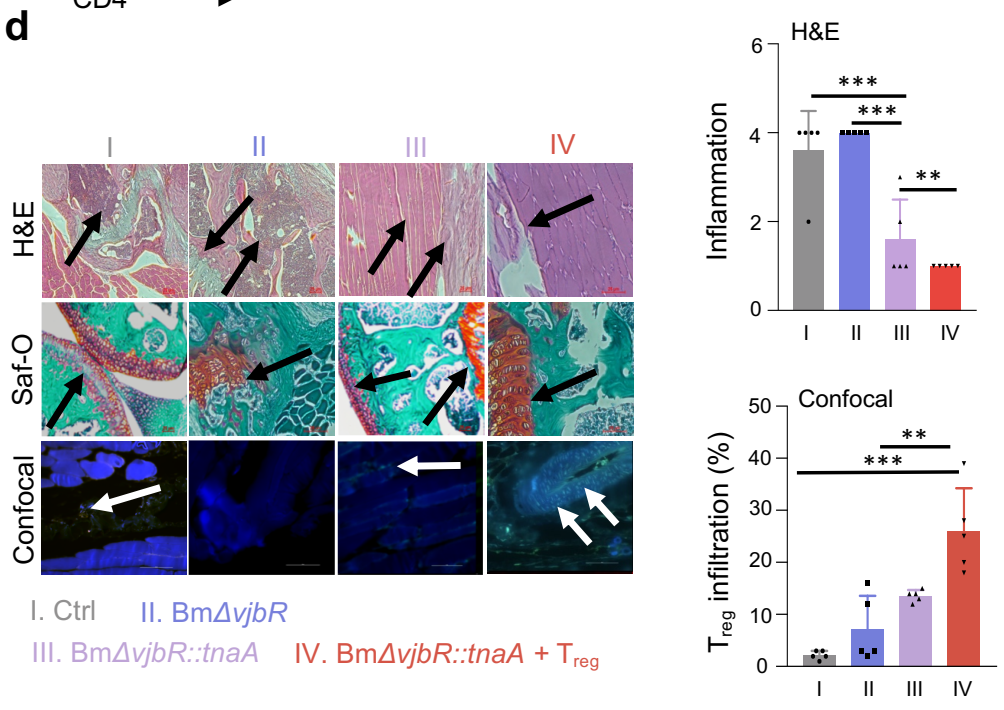

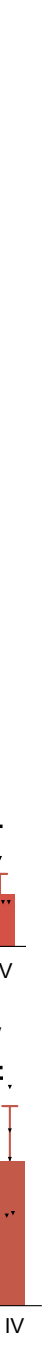


Fig. 2: $\mathrm{Bm} \triangle v j b R:$ tnaA significantly dampens inflammation and reduces arthritis in murine CIA model which is augmented by adoptive cell transfer (ACT) of $\mathrm{T}_{\text {regs. }} \mathrm{a}$, Cytokine arrays were used to measure pro-inflammatory cytokines produced by control, Bm $\Delta v j b R$, and $\mathrm{Bm} \Delta v j b R:$ tnaA treated BMDMs. b, BMDMs were treated with either $\mathrm{Bm} \Delta v j b R:: \operatorname{tn} A A$ or $\mathrm{Bm} \Delta v j b R$ and then co-cultured with T cells derived from pooled LNs and spleen of C57BL/6 mice. Flow cytometric assays were then performed to measure IFN- $y$ and TNF- $\alpha$ of T cells. c, Arthritis score and arthritis incidence in CIA C57BL/6 mice from control (Ctrl, gray bar); Bm $\Delta v j b R$ (blue bar); $B m \Delta v j b R:$ tnaA (pink bar) and $B m \Delta v j b R::$ tnaA followed by $A C T$ of $T_{\text {regs }}\left(T_{\text {reg }}\right.$, red bar) $(N=5$ in each group). d, Representative images of H\&E, Saf-O staining, and confocal microscopy from mouse knees on day 60 post CIA induction. Quantitative analysis of $\mathrm{T}_{\text {reg }}$ infiltration and inflammation scores from these mice are also shown. e, Cells from the LNs and spleen were collected from CIA-induced mouse groups (Ctrl, $\mathrm{Bm} \Delta v j b R:: t n a A$, and $\mathrm{Bm} \Delta v j b R:: \operatorname{tn} a A$ combined with ACT of $\mathrm{T}_{\text {regs. }}$. These cells were then stained and quantified by flow cytometry using markers for $\mathrm{CD}^{+}{ }^{+} \mathrm{T}$ cells and intracellular staining of FoxP3 $\left(\mathrm{T}_{\text {regs }}\right) . \mathbf{f}, \mathrm{ClA}$-induced mice were treated with PBS (Ctrl), ACT of $\mathrm{T}_{\text {regs }}$ only ( $\mathrm{T}_{\text {regs }}$ only; $\mathrm{N}=5$ ), or $\mathrm{Bm} \Delta v j b R:$ :tnaA combined with ACT of $\mathrm{T}_{\text {regs }}$ $(\mathrm{N}=5)$. Cells from the knee and ankle joints were stained with 21 markers and measured by CyTEK aurora flow cytometry. Heatmap shows immune cell profiles in different treatment groups of mice (scale bar represents percentage of cell in each treatment group within each cell type). g, viSNE map shows the four subtypes of B cells differentially expressed in the treated group of mice. Data represent means \pm SD. Student's $t$-test or Tukey's multiple comparisons test was applied for statistical analysis. ${ }^{*},{ }^{* *},{ }^{* * *}$ : significance at $p<0.05,0.01,0.001$. 
a

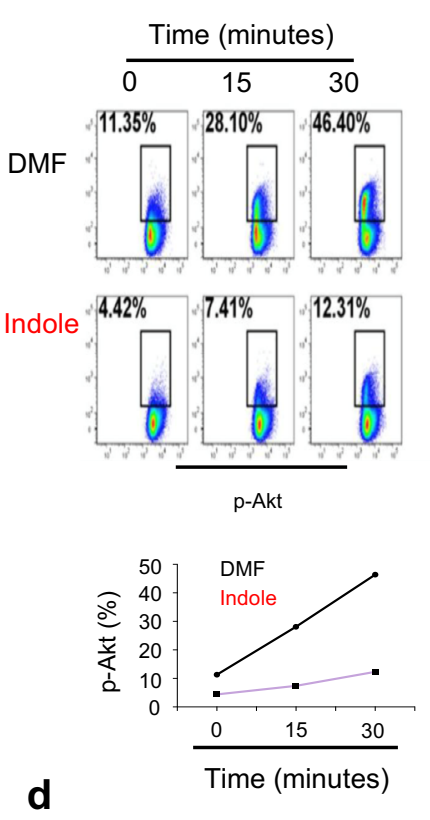

b
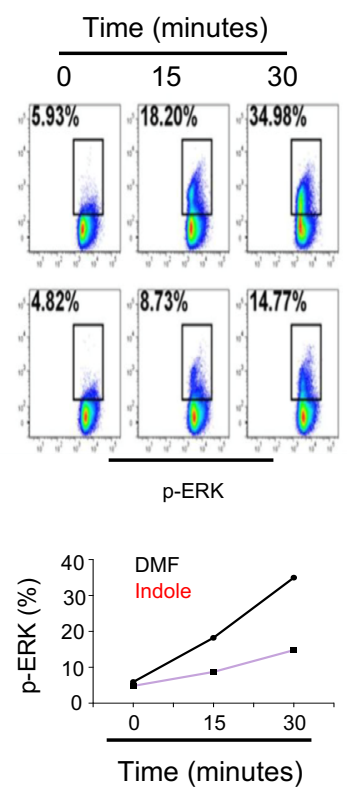

e
C

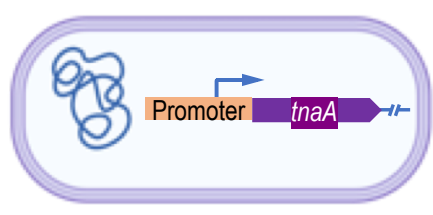

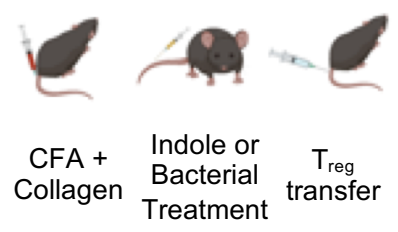

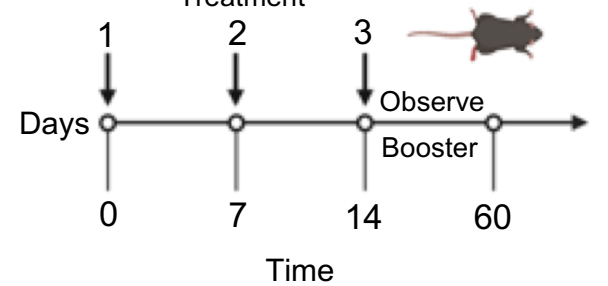

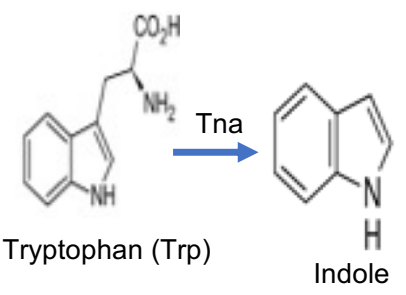

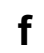

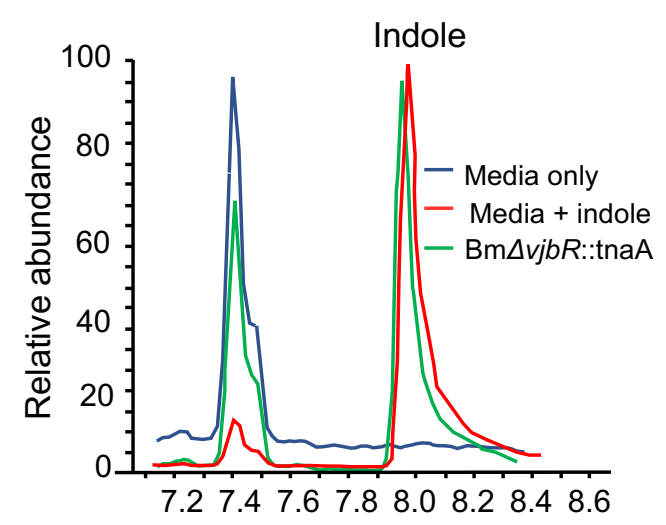

RT (min)

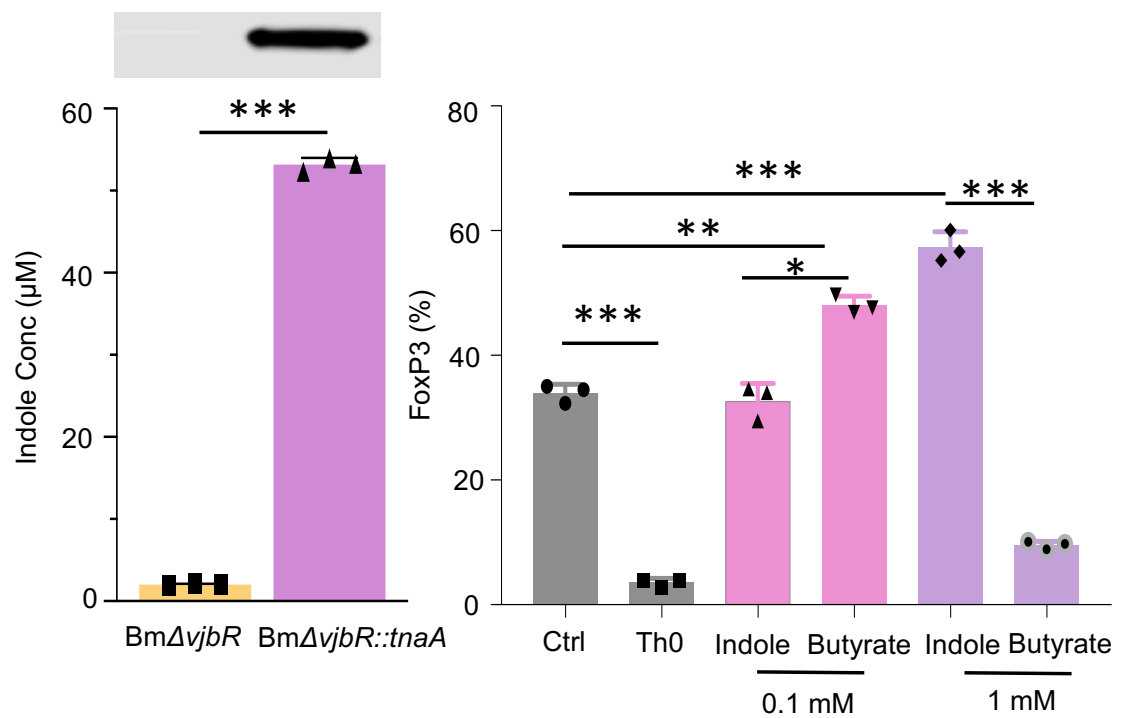




\section{Fig. S1: Indole suppresses immune cell activation and $B m \Delta v j B R$ is engineered to produce indole.}

a, Indole (1mM) suppresses the Akt and ERK signaling pathways in CD11 $\mathrm{b}^{+}$cells in different post incubation time. Flow cytometric dot-plot analysis and graphical representation of Akt and ERK expression with time. $\mathbf{b}$, Schematic diagram of murine CIA model used in all the subsequent studies. c, Schematic representation of the engineered $\mathrm{Bm} \Delta v j b R::$ tnaA harboring a plasmid carrying a tnaA expression cassette. The indole biosynthesis pathway is depicted in the figure. TnaA catalyzes the conversion of tryptophan to indole. d, Engineered $B m \Delta v j b R:: t n a A$ produced high concentration of indole as shown in the mass-spectrometric analysis. e, Western blotting analysis of the expression of tnaA protein in the parental strain compared with the engineered $\mathrm{Bm} \Delta v j b R:$ :tnaA strain. Graphical representation of the comparative analysis of indole production by $B m \triangle v j B R$ parental bacterial strain and the engineered $B m \Delta v j b R:$ tnaA strain. f, Indole promotes significantly higher expansion of $\mathrm{T}_{\text {regs }}$ compared to butyrate at $1 \mathrm{mM}$ concentration as shown in the graphical analysis. Graphical data derived from flow cytometric representatives as shown in Fig. 1C. Data represent means \pm SD. Student's $t$-test or Tukey's multiple comparisons test was applied for statistical analysis. ${ }^{*},{ }^{* *},{ }^{* * *}$ : significance at $p<0.05,0.01,0.001$. 


\section{a}
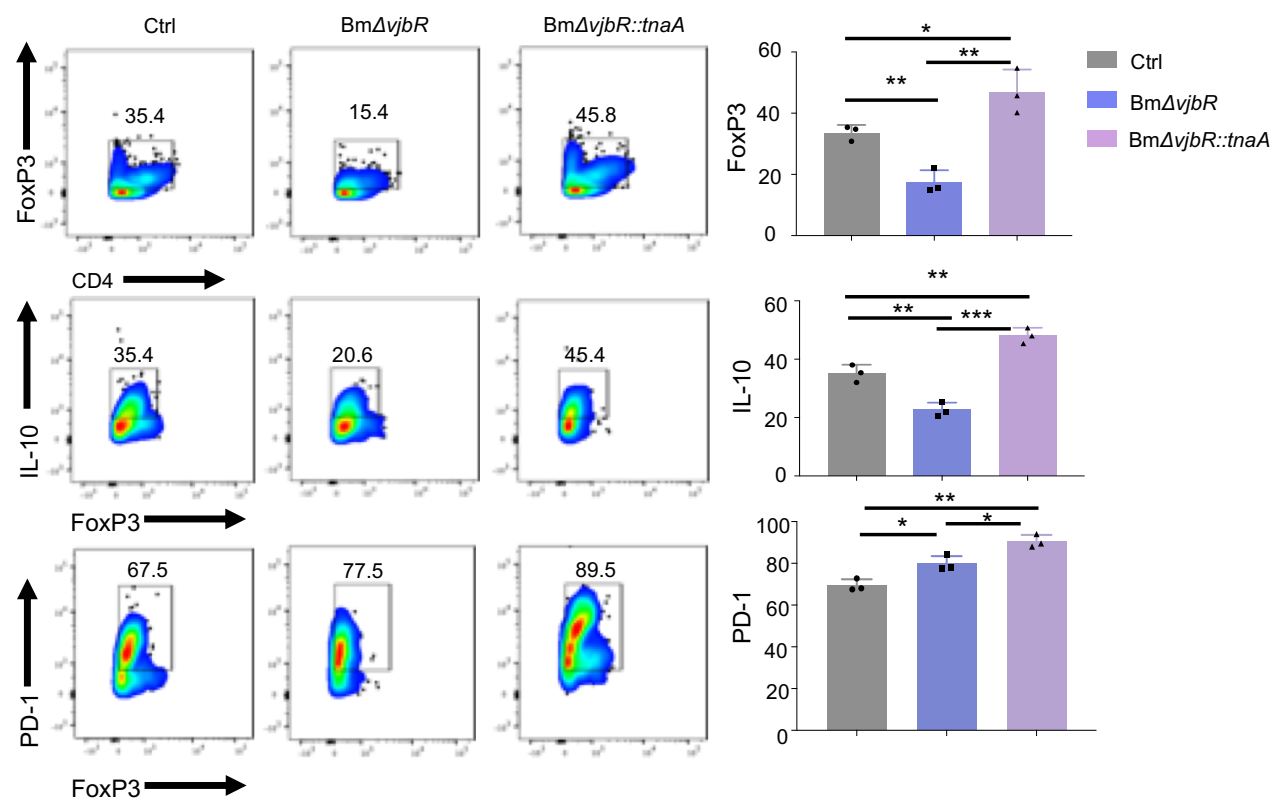

b

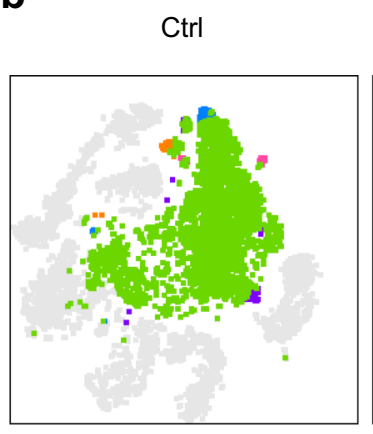

$T_{\text {reg }}$

$\mathrm{Bm} \Delta v j b R:: t n a A+\mathrm{T}_{\text {reg }}$
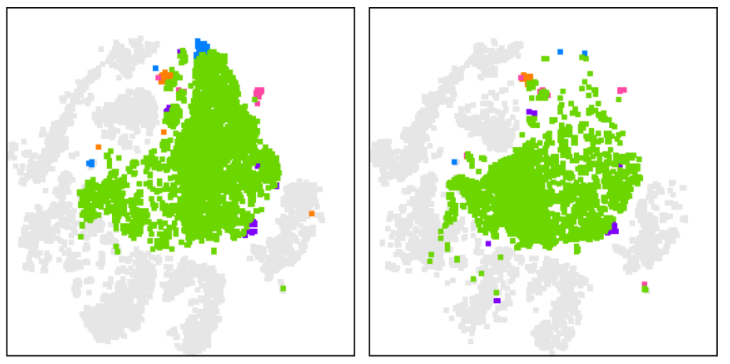
Fig. S2: Bm $\Delta v j b R:: t n a A$ activates $\mathrm{T}_{\text {regs }}$ and suppresses $\mathrm{B}$-cell mediated inflammation.

a, BMDMs infected with either $\mathrm{Bm} \Delta v j b R \cdot: \operatorname{tnaA}$ or $\mathrm{Bm} \Delta v j b R$ were co-cultured with $\mathrm{CD} 4^{+} \mathrm{T}$ cells from mouse LNs and spleen and activated by using anti-CD3/CD28 Abs. Flow cytometric dot-plot assay shows that $\mathrm{Bm} \Delta v j b R:$ :tnaA treated BMDMs greatly promoted expression of FoxP3 and PD-1 and production of IL-10 in CD4+ T cells. The dot-plots are followed by graphical representation from 3 independent experiments. b, The total CD19 population is shown in the viSNE plots. Data represent means \pm SD. Student's $t$ test or Tukey's multiple comparisons test was applied for statistical analysis. ${ }^{*},{ }^{* *},{ }^{* * *}$ : significance at $p<0.05,0.01,0.001$. 


\section{Table S1}

\begin{tabular}{|c|c|c|c|c|}
\hline Population & Cell type & Ctrl & $T_{\text {reg }}$ & $\begin{array}{c}\mathrm{Bm} \Delta v j b R \\
:: t n a A+ \\
\mathrm{T}_{\text {reg }} \\
\end{array}$ \\
\hline 1 & CD40L hi CD25 lo & $8.6 \%$ & $18.7 \%$ & $72.7 \%$ \\
\hline 2 & CD25 hi Tim3 hi Ki67 hi & $34.9 \%$ & $20.4 \%$ & $44.7 \%$ \\
\hline 3 & D45 lo CD44 mid & $34.7 \%$ & $22.2 \%$ & $43.1 \%$ \\
\hline 4 & CD45 lo CD44 lo & $34.3 \%$ & $27.9 \%$ & $37.8 \%$ \\
\hline 5 & CD44 hi CD160 mid & $28.0 \%$ & $36.0 \%$ & $36.0 \%$ \\
\hline 6 & CD3 hi CD8hi CD25 lo & $31.8 \%$ & $33.3 \%$ & $34.9 \%$ \\
\hline 7 & CD19 hi CD62L lo & $32.7 \%$ & $33.6 \%$ & $33.7 \%$ \\
\hline 8 & CD4 hi CD25 lo & $31.9 \%$ & $36.8 \%$ & $31.3 \%$ \\
\hline 9 & CD4 hi CD25 mid CTLA4 lo & $37.0 \%$ & $32.0 \%$ & $31.0 \%$ \\
\hline 10 & CD25 hi PD-L1 hi & $47.1 \%$ & $22.9 \%$ & $30.0 \%$ \\
\hline 11 & CD8 hi CD62L lo & $38.2 \%$ & $32.4 \%$ & $29.4 \%$ \\
\hline 12 & CD19 hi CD40L hi & $40.6 \%$ & $36.7 \%$ & $22.7 \%$ \\
\hline 13 & CD19 hi PD-1 mid CTLA4 hi & $46.6 \%$ & $32.3 \%$ & $21.1 \%$ \\
\hline 14 & CD19 hi CD40L hi & $32.1 \%$ & $47.4 \%$ & $20.5 \%$ \\
\hline 15 & CD25 hi CD140 hi & $20.1 \%$ & $60.3 \%$ & $19.6 \%$ \\
\hline 16 & CCR7 hi PD-1 lo & $53.1 \%$ & $28.0 \%$ & $18.9 \%$ \\
\hline 17 & CD4 mid PD-L1 hi & $55.2 \%$ & $29.3 \%$ & $15.5 \%$ \\
\hline 18 & CD19 hi PD-1 lo CD40L lo & $76.0 \%$ & $22.3 \%$ & $1.68 \%$ \\
\hline
\end{tabular}

Supplementary Table 1. A list of different immune cell types which were differentiated using the tSNE analysis and their proportion in different treatment groups. 


\begin{tabular}{|l|l|l|l|}
\hline Ab & Clone & Fluorophore & Source \\
\hline Ghost Dye Red 710 & & Ghost Dye Red 710 & Tonbo Biosciences \\
\hline CD45 & 30-F11 & FITC & Tonbo Biosciences \\
\hline CD8a & $53-6.7$ & BUV395 & BD Biosciences \\
\hline CD4 & GK1.5 & eFluor 450 & Thermo Fisher Scientific \\
\hline CD3 & 17 2 & APC-Fire 810 & BioLegend \\
\hline CD19 & 1D3 & BUV805 & BD Biosciences \\
\hline MHC II & M5/114.15.2 & Violet Fluor 500 & Tonbo Biosciences \\
\hline CD160 & 7H1 & APC & BioLegend \\
\hline PD-L1 & MIH5 & Super Bright 780 & Thermo Fisher Scientific \\
\hline CTLA-4 & UC10-4F10-11 & PE-Cy7 & Tonbo Biosciences \\
\hline PD-1 & 29F.1A12 & PE-Dazzle 594 & BioLegend \\
\hline Lag-3 & C9B7W & BUV661 & BD Biosciences \\
\hline TIM-3 & 5D12/TIM-3 & Brilliant Violet 605 & BD Biosciences \\
\hline CD44 & IM7 & PerCP & BioLegend \\
\hline CD62L & HRL1 & BUV737 & BD Biosciences \\
\hline Ki-67 & B56 & Brilliant Violet 711 & BD Biosciences \\
\hline CCR7 & $4 B 12$ & PE & BioLegend \\
\hline CD69 & H1.2F3 & BUV563 & BD Biosciences \\
\hline CD25 & PC61 & Brilliant Violet 650 & BioLegend \\
\hline CD40L & MR1 & BB700 & BD Biosciences \\
\hline CD28 & 37.51 & BUV496 & BD Biosciences \\
\hline & & & \\
\hline
\end{tabular}

Supplementary Table 2: List of Abs used in CyTEK assay 


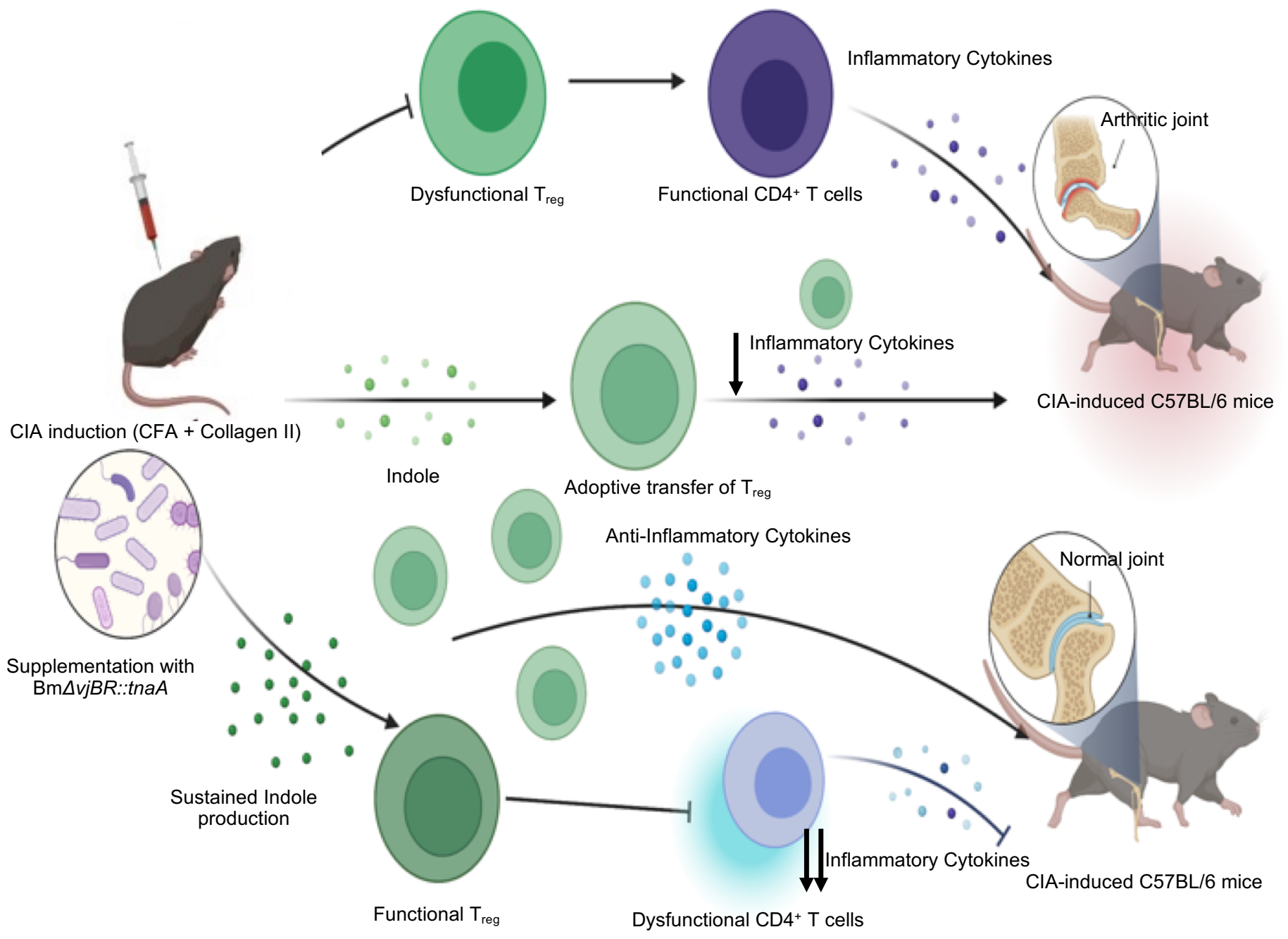




\section{Schematic representation of amelioration of CIA in C57BL/6 mice}

The illustration depicts the process of amelioration of CIA in C57BL/6 mice by the engineered $B m \Delta v j b R:: t n a A$ bacterial strain. $C 57 \mathrm{BL} / 6$ mice may harbor a microbiome deficient in production of the metabolite indole. When challenged with the CIA, these mice rapidly develop arthritis due to dysfunctional $\mathrm{T}_{\text {regs }}$ and rapid expansion of CD4 ${ }^{+}$ effector $\mathrm{T}$ cells. However, when $B m \Delta v j b R:: t n a A$ bacteria are administered to mice bearing CIA the $T_{\text {reg }}$ activity is substantially increased and the pathogenic activity of $\mathrm{CD}^{+}$effector $\mathrm{T}$ cells is compromised, which results in amelioration of joint inflammation and improved synonyms. This activity is greatly augmented by adoptive transfer of $T_{\text {regs. }}$. 